\title{
Single-Atom Quantum Probes for Ultracold Gases Boosted by Nonequilibrium Spin Dynamics
}

\author{
Quentin Bouton, ${ }^{1}$ Jens Nettersheim, ${ }^{1}$ Daniel Adam, ${ }^{1}$ Felix Schmidt, ${ }^{1}$ Daniel Mayer, ${ }^{1}$ \\ Tobias Lausch, ${ }^{1}$ Eberhard Tiemann $\odot,{ }^{2}$ and Artur Widera $\oplus^{1,3, *}$ \\ ${ }^{1}$ Department of Physics and Research Center OPTIMAS, Technische Universität Kaiserslautern, \\ 67663 Kaiserslautern, Germany \\ ${ }^{2}$ Institut für Quantenoptik, Leibniz Universität Hannover, 30167 Hannover, Germany \\ ${ }^{3}$ Graduate School Materials Science in Mainz, Gottlieb-Daimler-Strasse 47, \\ 67663 Kaiserslautern, Germany
}

(Received 11 July 2019; revised manuscript received 23 October 2019; published 27 January 2020)

\begin{abstract}
Quantum probes are atomic sized devices mapping information of their environment to quantummechanical states. By improving measurements and at the same time minimizing perturbation of the environment, they form a central asset for quantum technologies. We realize spin-based quantum probes by immersing individual $\mathrm{Cs}$ atoms into an ultracold $\mathrm{Rb}$ bath. Controlling inelastic spin-exchange processes between the probe and bath allows us to map motional and thermal information onto quantum-spin states. We show that the steady-state spin population is well suited for absolute thermometry, reducing temperature measurements to detection of quantum-spin distributions. Moreover, we find that the information gain per inelastic collision can be maximized by accessing the nonequilibrium spin dynamic. Keeping the motional degree of freedom thermalized, individual spin-exchange collisions yield information about the gas quantum by quantum. We find that the sensitivity of this nonequilibrium quantum probing effectively beats the steady-state Cramér-Rao limit by almost an order of magnitude, while reducing the perturbation of the bath to only three quanta of angular momentum. Our work paves the way for local probing of quantum systems at the Heisenberg limit, and moreover, for optimizing measurement strategies via control of nonequilibrium dynamics.
\end{abstract}

DOI: 10.1103/PhysRevX.10.011018

\section{INTRODUCTION}

Miniaturizing measurement probes is a strong technological driving force and yields fascinating new insights into various fields including biology [1], solid-state physics [2], and metrology [3]. A fundamental limit of miniaturization is the use of single atoms as individual probes, opening the door to employing quantum properties for advanced probing. A paradigm for quantum probing is a single atom with discrete energy quantum levels coupled to an atomic environment. Extracting relevant information stored in quantum levels of the probe can enhance the information obtained about a (quantum) environment under investigation. At the same time, the unavoidable perturbation of the environment caused by the measurement process can be reduced. The potential of quantum probes has been

*widera@physik.uni-kl.de

Published by the American Physical Society under the terms of the Creative Commons Attribution 4.0 International license. Further distribution of this work must maintain attribution to the author(s) and the published article's title, journal citation, and DOI.
Subject Areas: Atomic and Molecular Physics, Quantum Physics the focus of intense recent theoretical studies [4-6], with a strong emphasis on quantum thermometry. In classical thermometry, a thermometer thermalizes with the bath, and the mean kinetic energy of the probe is taken as a measure for the bath temperature presuming a Maxwell-Boltzmann distribution (Fig. 1). Thermometry of quantum systems is particularly important for ultracold gases, and various probes including magnons [7], confined Bose-Einstein condensate [8], Fermi sea [9], or single atoms [10] have been reported. All these probes rely on the standard method of time-of-flight velocimetry [11] and thus are classical. Exploiting the quantum properties of probes, however, has been shown to enhance precision and sensitivity, being ultimately limited by the Cramér-Rao relation [12]. Numerous schemes have thus been proposed to extract temperature or work distributions via quantum probing [5,13-17]. The experimental demonstration of probing an atomic gas using the quantum properties of individual atoms, however, is so far elusive. Here, we show the control of the microscopic mechanism of motion-spin coupling, which is the key to our single-atom quantum probe. Moreover, having access to the dynamics of this microscopic process of quantum probing allows us to optimize 


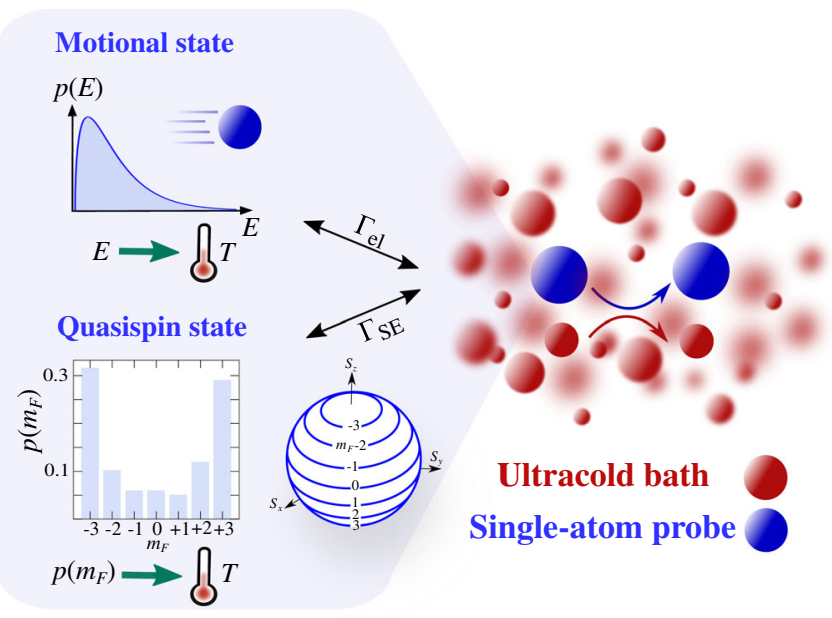

FIG. 1. Single-atom quantum probing of an ultracold gas. A single atom is coupled to the bath, interacting via two processes. First, elastic collisions at rate $\Gamma_{\mathrm{el}}$ thermalize the probe to the temperature of the bath, where the kinetic energy distribution allows for classical thermometry (top). Second, spin-exchange collisions at rate $\Gamma_{\mathrm{SE}}$ ensure motion-spin mapping and allow us to store information on the bath energy in the quantum states of the probe, here the quasispin states visualized by a macroscopic Bloch sphere (bottom).

the information obtained from the probe, boosting the sensitivity of the probe beyond fundamental bounds of steady-state systems using nonequilibrium dynamics.

\section{EXPERIMENTAL REALIZATION OF SPIN QUANTUM PROBES}

We realize such a quantum probe by mapping the information about the bath onto the quantum states of single atoms. Experimentally, we use the discrete quasispin levels of a single cesium (Cs) atom for quantum probing. The Cs probes are immersed into an ultracold gas of rubidium $(\mathrm{Rb})$ and allow us to extract the temperature of the bath as well as the absolute value of the surrounding magnetic field. Moreover, we show that the sensitivity can be significantly enhanced by considering nonequilibrium spin dynamics of the quantum probe.

The standard approach proposed for quantum probing is mapping of thermal information onto vibrational states of trapped particles [18] such as neutral atoms in optical tweezers [19] or trapped ions [20]. Our approach of using quasispin states is particularly suited for ultracold temperatures and at the same time allows us to independently control the trapping parameters. The relevant energy scales of our quantum probe are the thermal energy $k_{B} T$, with $k_{B}$ the Boltzmann constant, and the magnetic energy of the probe's Zeeman levels in a weak magnetic field $\Delta E / 2=$ $g_{F} \mu_{B} B$, where $g_{F}$ is the Landé factor and $\mu_{B}$ the Bohr magneton. For a magnetic field of $B=10 \mathrm{mG}$, the energy splitting corresponds to $g_{F} \mu_{B} B / k_{B} \sim 170 \mathrm{nK}$. For comparison, this energy corresponds to a trap-level spacing of
$3.5 \mathrm{kHz}$, which is well below the values of vibrational-level spacing for tight traps.

Individual laser-cooled $\mathrm{Cs}$ atoms are initially prepared in the Zeeman state $\left|F_{\mathrm{Cs}}=3, m_{F, \mathrm{Cs}}=2\right\rangle$, where $F_{\mathrm{Cs}}$ is the total angular momentum and $m_{F, \mathrm{Cs}}$ its projection on the quantization axis. The $\mathrm{Rb}$ bath is prepared in $\left|F_{\mathrm{Rb}}=1, m_{F, \mathrm{Rb}}=0\right\rangle$ with temperatures ranging from $T=$ 0.2 to $1 \mu \mathrm{K}$ [21], for details see Appendix A. Interaction between the probe atom and bath is initiated by transporting the $\mathrm{Cs}$ atom into the $\mathrm{Rb}$ cloud and comprises two processes (Fig. 1). First, frequent elastic collisions at rate $\Gamma_{\mathrm{el}}$ between the probe atom and bath ensure thermalization of the probe's motional degree of freedom (d.o.f.) with the bath, while leaving the internal states unaffected. Second, motion-spin mapping occurs via inelastic spin-exchange (SE) collisions at rate $\Gamma_{\mathrm{SE}} \approx \Gamma_{\mathrm{el}} / 10$ estimated for the present experimental conditions. SE collisions exchange individual quanta of angular momentum between the probe and bath, where the Zeeman energy shifts for Rb and Cs differ by a factor of 2 due to $g_{F, \mathrm{Rb}}=2 g_{F, \mathrm{Cs}}$. Two types of SE can occur: exoergic SE, which has been studied, for example, in Ref. [22], and endoergic SE (Fig. 2), which opens as an additional new process when the Zeeman energy becomes comparable to the thermal energy of the bath. In fact, endoergic processes contribute the desired information on the thermal energy. For exoergic (endoergic) SE, an energy of $\Delta E / 2$ is released (lacking) between the initial and final states of the Cs- $\mathrm{Rb}$ collision partners while changing the atomic quasispin accordingly (see Fig. 2). Exoergic processes are thus always allowed and tend to drive the probe's spin population toward $m_{F, \text { Cs }}=-3$. By contrast, endoergic processes can occur only if the missing energy difference of Zeeman states can be provided by the kinetic, and thus thermal, energy for the collisional process. This discrimination of SE by thermal energy is the microscopic mechanism of motion-spin mapping and effectively cools the collision partners similar to Pomeranchuck cooling [23]. The mechanism also prevails in dipolar gases $[24,25]$ converting kinetic energy of the gas into magnetic energy, and it is used to cool the entire sample. In our situation, in both SE processes, frequent elastic collisions quickly rethermalize the probe well before the next SE collision. The values of the SE rates depend on the atomic states as well as the collisional energy and can be precisely modeled [22], see Appendix C. Important insight into the quantum probing can already be obtained from a purely energetic threshold argument. The fraction of atoms that are energetically allowed to undergo an endoergic collision is given by

$$
p(B, T)=\int_{\Delta E(B) / 2}^{\infty} p\left(E_{c}\right) d E_{c},
$$

assuming a Maxwell-Boltzmann distribution $p\left(E_{c}\right)$ of collision energies $E_{c}$ (Fig. 2), for details see Appendix B. Therefore, modifying the relative 
(a)

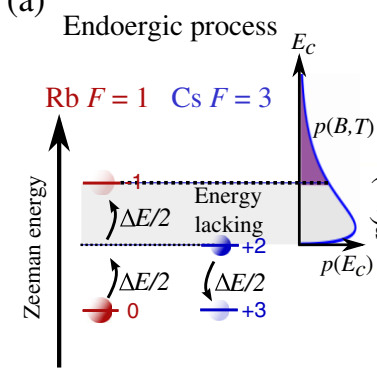

(b)

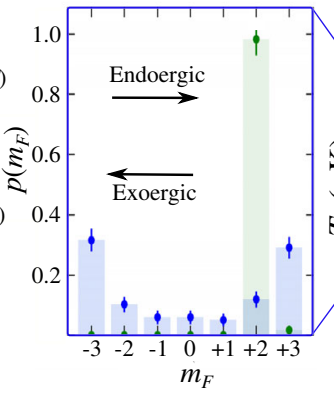

(c)

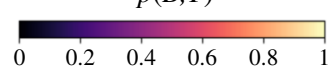

(d)

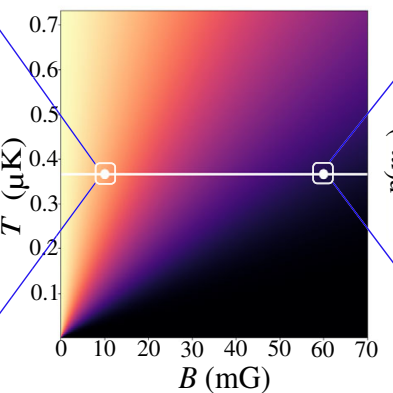

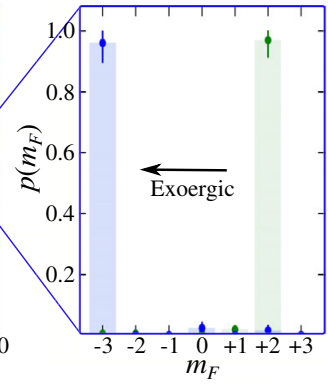

(e) Exoergic process

FIG. 2. Mechanism of motion-spin mapping onto quantum states. (a) Sketch of the endoergic process. Cs atoms go to a lower magnetic substate and release $\Delta E / 2$ of energy, whereas $\mathrm{Rb}$ atoms go to a higher magnetic substate and require $\Delta E$ of energy. Therefore, this process can occur only if the missing Zeeman energy can be provided by kinetic energy $E_{c}$ during the collision, which is only possible for a fraction $p(B, T)$ of the probe atoms. (b) Experimental spin population of Cs atoms for a magnetic field of $B=10 \mathrm{mG}$ before (green) and after 350-ms interaction time in a Rb bath at $366_{-40}^{+60} \mathrm{nK}$ (blue). We measure a nonzero population in $m_{F, \mathrm{Cs}}=+3$ (endoergic SE) and $m_{F, C s}=-3$ (exoergic SE). (c) Fraction of Cs atoms allowing to undergo an endoergic process as a function of the magnetic field $B$, assuming a Maxwell-Boltzmann distribution at temperature $T$. The indicated values correspond to measurements in (b) $p(B, T)=0.8$ and (d) $p(B, T)=0.1$. (d) Same as (b) but for $B=60 \mathrm{mG}$. Here, exoergic processes dominate, yielding a measured population (in blue) of Cs atoms in $m_{F, \mathrm{Cs}}=-3$. (e) Sketch of the exoergic process. $\mathrm{Rb}$ atoms are promoted to a lower magnetic state and release $\Delta E$, while Cs atoms are left in a higher magnetic state and need only $\Delta E / 2$. As a consequence, this process has no energetic threshold and releases $\Delta E / 2$ of energy into the system.

contributions of Zeeman and thermal energies allows us to microscopically tune the probability for an endoergic collision. Hence, the Cs spin distribution and its dynamics reflect precisely the competition between magnetic and thermal energies via the probability of endoergic collisions. In fact, any additional mechanism shifting the total energy of an atomic collision can also be sensed by our atomic quantum probe.

\section{RESULTS}

The ensuing time evolution of our quantum probe's spin population is shown in Fig. 3(a) together with the projected steady-state spin distribution in the background. We observe a redistribution of the probe's spin population over time toward the steady state, due to the competition of the rates between the exoergic and endoergic processes. To reach this state, the probe has to undergo a dozen SE collisions. Each $\mathrm{SE}$ collision also modifies the spin state of one $\mathrm{Rb}$ atom. The strong imbalance between the probe and the bath and the relatively short interaction time imply that the assumption of an ideal Markov bath applies here; i.e., in every SE collision the probe interacts with a $\mathrm{Rb}$ atom in the initial quantum state $m_{F, \mathrm{Rb}}=0$. We model the time evolution of the probe's spin population with a full rate model. All SE processes are integrated based on high-precision data at ultralow atomic collision energies obtained in previous work [22]. In short, the SE collision rates $\Gamma=\langle n\rangle \sigma \bar{v}$ of the model are directly inferred from atomic cross sections $\sigma$, where $\bar{v}$ is the relative velocity between $\mathrm{Rb}$ and $\mathrm{Cs}$, and $\langle n\rangle$ their density overlap, both calculated assuming thermalized atoms. The different values of the scattering cross sections $\sigma$ and their dependence on temperature $T$ and magnetic field $B$ are based on a precise model of the $\mathrm{Rb}$-Cs molecular potential $[22,26]$, which is in detail discussed in Appendix D. Our rate model fully captures the spin dynamics and yields excellent agreement for the time evolution of the probe's spin population for all parameters; an example is given in Fig. 3(a).

\section{A. Absolute thermometry}

Absolute bath thermometry can be performed using the probe's steady-state quasispin distribution. For the limiting case approaching $T=0$, endoergic processes are absent, and the steady state is a polarized state of the probe in $m_{F, \mathrm{Cs}}=-3$. For increasing temperature, endoergic processes emerge, leading to a spread of the quantum probe's steady-state spin population. We thus theoretically investigate the fluctuations of the energy associated with the probe's steady-state spin population $\sigma_{E}^{2}=\left\langle E^{2}\right\rangle-\left\langle E^{2}\right\rangle$ for different bath temperatures shown in Fig. 3(b). We find a linear increase of the spin distribution's width with bath temperature, see Appendix F, where the proportionality constant is independent of the specific magnetic field value as shown in the inset of Fig. 3(b), also of the initial state of the probe, $\mathrm{Rb}$ densities, and number of spin collisions since we consider here the steady state. In this case, the rates and thus the steady-state spin distribution depend only on the scattering cross sections. Hence, our quantum probe is well suited for absolute thermometry, allowing us to extract temperature information from spin-population measurements at known magnetic field values. 


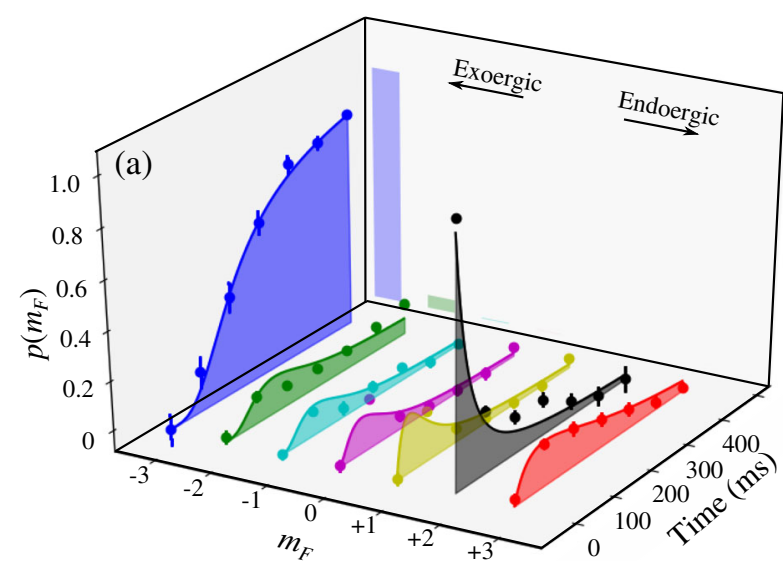

(b)

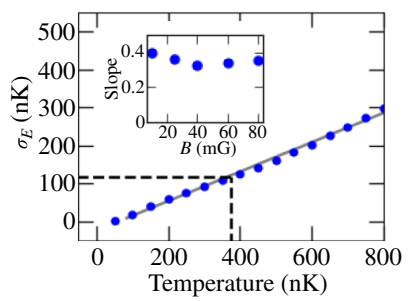

(c)

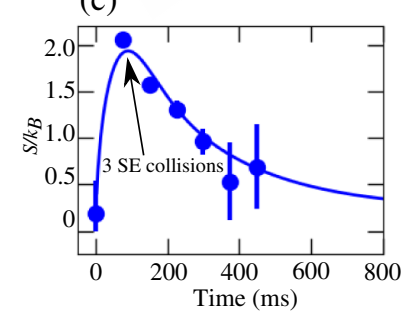

FIG. 3. Information gain from the spin distribution of quantum probes. (a) Quasispin-state dynamics of Cs atoms immersed in a $\mathrm{Rb}$ bath at $\mathrm{T}=366_{-40}^{+60} \mathrm{nK}$ at a fixed magnetic field $B=25 \mathrm{mG}$ [expected endoergic fraction $p(B, T)=0.54$ ]. Experimental time trace (dots) and theoretical predictions of our rate model (solid line, for details see Appendix D) are shown. Each experimental point is an average over approximately 200 measurements, and the error bars indicate the statistical uncertainties in the atom number determination. The histogram projected in the back plane shows the steady-state spin distribution. (b) Theoretically calculated probe-energy fluctuations $\sigma_{E}^{2}$ of the steady state for $B=$ $25 \mathrm{mG}$ calculated from modeled quantum-state distributions as $\left\langle E^{2}\right\rangle=\sum_{m_{F}} E_{m_{F}}^{2} P_{m_{F}}$, with $E_{m_{F}}$ and $P_{m_{F}}$ being the energy and population probability of quantum state $m_{F}$. The $P_{m_{F}}$ are the steady-state solutions of our rate model. Dashed lines direct to $T=366 \mathrm{nK}$. Inset: Slope of the linear trend between $\sigma_{E}$ and $T$ for different values of the magnetic field. (c) Time evolution of the probe entropy calculated from the spin populations of (a). The time of maximal entropy corresponds to an average of three SE collisions ( 0.5 endoergic and 2.5 exoergic).

Importantly, the steady state observed is not the equilibrium state of the total system. Indeed, each SE collision also modifies the spin state of one $\mathrm{Rb}$ atom while preserving the total magnetization of the system. The probe's steady state studied here corresponds to a timescale where in every SE process, the probe interacts with a $\mathrm{Rb}$ atom in the initial quantum state; i.e., the assumption of an ideal Markov bath applies. However, although the Cs spin distribution is in steady state, SE collisions will continue to modify the spin states of Rb atoms. At a longer timescale, after many more SE collisions (approximately $N_{\mathrm{Rb}}$ ), the $\mathrm{Rb}$ bath's spin population will be significantly modified. Because of the strong imbalance of quantum probes and gas atoms, the Cs spin distribution can adapt to small changes of the $\mathrm{Rb}$ spin distribution on a much faster timescale and will thus always remain approximately in steady state, where the specific steady-state distribution depends on the actual $\mathrm{Rb}$ state. In this limit, the assumption of a Markov bath is not fulfilled anymore. We expect the system to eventually reach an equilibrium state, where the conservation of total energy and magnetization suggest the description by a Gibbs ensemble.

\section{B. Nonequilibrium impurity quantum probing}

While steady-state thermometry yields information that is independent of the details of the interaction, experimentally it features several drawbacks. First, atom loss can prevent long interaction times, especially for large bath densities. Second, albeit the number of SE collisions is small compared to the number of atoms in the gas, identifying the least-perturbative measurement protocol for quantum probing is of fundamental interest. We therefore investigate the information obtained during the nonequilibrium time evolution of the probe's spin distribution. To quantify the information gain per SE collision, we use the Shannon entropy [27] of the quantum probe's spin distribution which is given by

$$
S=-k_{B} \sum_{m_{F}} P_{m_{F}} \log \left(P_{m_{F}}\right) .
$$

The time dependence of $S$ is plotted in Fig. 3(c). In our case, the entropy is maximized when the distribution is equally spread, as can be derived using Lagrange multipliers, see Appendix E. For our initial conditions, we find a maximum of the entropy for a mean of only three SE collisions, where we come closest to this situation. It indicates that the nonequilibrium spin distribution can provide much more information than the steady-state distribution while at the same time reducing the bath perturbation.

We quantify the performance of the nonequilibrium probing by first considering the information obtained from finite-time data taken at an interaction time of $350 \mathrm{~ms}$ (Fig. 4). We perform thermometry or magnetometry by varying the bath temperature or magnetic field value, leaving the respective other value fixed. The nonequilibrium values for the temperature $\left(T_{\text {spin }}\right)$ or magnetic field $\left(B_{\text {spin }}\right)$ are determined by comparing the measured quasispin populations with our numerical model using a $\chi^{2}$ analysis [28], where only $T_{\text {spin }}$ or $B_{\text {spin }}$ is a free parameter, see Appendix G. We compare the quantum probe's values with independently measured values of the time-of-flight velocimetry for the temperature and microwave spectroscopy of $\mathrm{Rb}$ hyperfine transitions for the magnetic field and find good agreement. While in general the spin d.o.f. is decoupled from the motional d.o.f. in quantum-gas experiments, endoergic SE allows controlled coupling, and thus, 

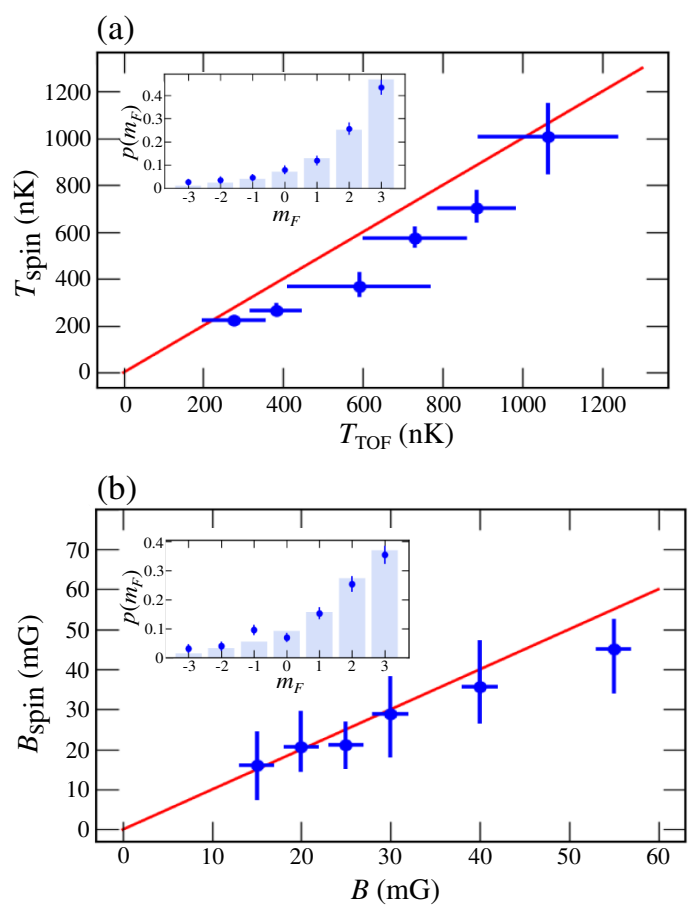

FIG. 4. Nonequilibrium quantum probing. (a) Comparison of the temperatures extracted from the spin population of Cs atoms $T_{\text {spin }}$ after 350-ms interaction time to time-of-flight temperatures of the $\mathrm{Rb}$ cloud $T_{\mathrm{TOF}}$ for a fixed magnetic field $B=10 \mathrm{mG}$. The error bars of $T_{\text {spin }}$ originate from the statistical errors on the $\chi^{2}$ analysis, and the error bars of $T_{\mathrm{TOF}}$ reflect the shot-to-shot fluctuations in the experiment (around 10\%-15\%). The red line serves as a guide to the eye $T_{\text {spin }}=T_{\text {TOF. Inset: Example of spin population. The dots }}$ represent the data and the histogram the theory with the best-fitting temperature, here $T_{\text {spin }}=1008_{-160}^{+140} \mathrm{nK}$. (b) Same as (a) but with the magnetic field $B$ for a fixed temperature $T=1 \mu \mathrm{K}$. Inset: Spin population with $B_{\text {spin }}=20.7_{-6.2}^{8.9} \mathrm{mG}$.

allows us to compare information from two very different measurement approaches.

Second, we investigate the sensitivity of the nonequilibrium probing, making use of the quantum Fisher information $F$ as an indicator of the thermal and magnetic sensitivities. Fisher information is a key concept in parameter estimation theory [29] and has been used to quantify many observables ranging from temperature to entanglement [6,30-32] and recently for cold atom magnetometry [33]. Neglecting coherence in the system, we describe each state by a diagonal density matrix $\hat{\rho}(B, T)=$ $\sum_{m_{F}} P_{m_{F}}(B, T)\left|m_{F}\right\rangle\left\langle m_{F}\right|$, where $P_{m_{F}}(B, T)$ are the spin populations of the probe at $T$ and $B$. We denote the parameter of interest as $\theta(\theta=B$ or $T)$. As discussed in Appendix $\mathrm{H}$, we quantify the distance between two quantum states at $\theta$ and $\theta+\delta \theta$ using the Bures distance as

$$
d_{\text {Bures }}^{2}(\delta \theta)=2-2 \sum_{m_{F}}\left[P_{m_{F}}(\theta) P_{m_{F}}(\theta+\delta \theta)\right]^{1 / 2},
$$

which coincides with the Hellinger distance [34] for commuting density operators. A Taylor expansion to first order of the Bures distance defines the usual connection between Bures distance and $F_{\theta}$,

$$
d_{\text {Bures }}(\delta \theta)=\sqrt{F_{\theta}} \delta \theta+\mathcal{O}(\delta \theta)^{2} .
$$

Hence, high sensitivities indicated by a large value of $F_{\theta}$ also imply a high statistical speed $\partial d_{\text {Bures }} / \partial \delta_{\theta}=\sqrt{F_{\theta}}$ to change the Bures distance according to the parameter change. Thus, we refer to $\sqrt{F_{\theta}}$ as sensitivity. In Figs. 5(a) and 5(b), we investigate the time evolution of the thermal $(\theta=T)$ and magnetic $(\theta=B)$ sensitivities of our quantum probe. We observe that the sensitivity reaches a maximum in both cases, which outperforms the steady-state sensitivity by a factor of 6.55 (17.5) for thermometry (magnetometry). This
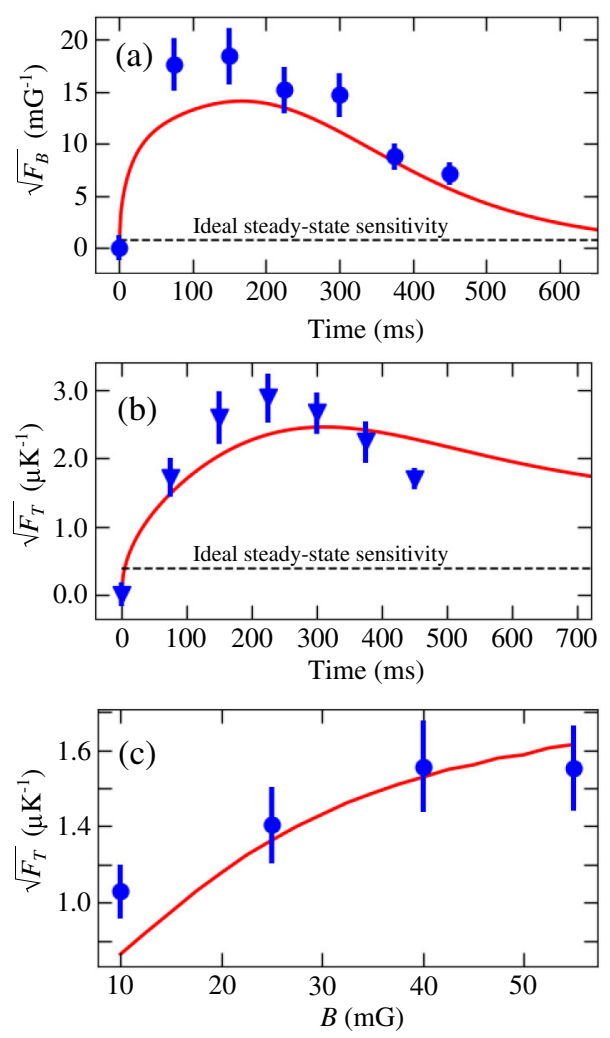

FIG. 5. Nonequilibrium boost of quantum-probe sensitivity. Time dependence of the magnetic sensitivity $\sqrt{F_{B}}$ (for $T=590 \mathrm{nK}$, centered at $B=40 \mathrm{mG}$ ) (a) and thermal sensitivity $\sqrt{F_{T}}$ (for $B=40 \mathrm{mG}$, centered at $T=640 \mathrm{nK}$ ) (b) of the quantum probe. Red lines correspond to theoretical calculations, blue dots or triangles are experimental data; dots are sensitivities extracted comparing only experimental populations, whereas triangles indicate sensitivities extracted comparing measured populations to theoretical ones. Dashed lines represent the respective sensitivity value expected for the steady state. (c) Thermal sensitivity (centered at $T=640 \mathrm{nK}$ ) as a function of the $B$ field for an interaction time that fixes the number of SE collisions to approximately 4. 
implies that nonequilibrium probing also outperforms the Cramér-Rao bound [12] of steady-state probing. In both cases, the optimal time of highest sensitivity time is close to the time where the entropy of the quantum probe's spin distribution is also maximum, i.e., where the amount of information gain is largest. This observation points toward further desirable experimental studies. In fact, in quantuminformation theory, Shannon entropy and Fisher information are key concepts and are subject to intensive theoretical studies [35]. Our system may shed new light on the connection between these two quantities.

In Fig. 5(c), we study the thermal sensitivity of the probe at fixed time adjusted to a constant number of 4.2(3) exoergic spin collisions. We observe that the sensitivity per collision increases with the magnetic field $B$. We explain this observation by the decrease in the amount of endoergic processes with an increase of $B$ : for $B=10 \mathrm{mG}$, the Cs atoms experience 2.3(2) endoergic spin collisions but only 0.5 (1) for $B=55 \mathrm{mG}$. For the large- $B$ field, the spin population is mainly driven by the exoergic process, and the state $m_{F}=-3$ is populated. The little fraction of endoergic process drastically changes the population from $m_{F}=-3$ to a redistribution toward the other states when the temperature $T$ is varied. This leads to a large sensitivity. At low magnetic field, the Cs population is already distributed among all the spin states due to higher endoergic spin-collision rates. Therefore, there is no drastic change in the spin population when $T$ changes, leading to a lower sensitivity.

\section{CONCLUSION}

The realization of individual atomic quantum probes yielding access to information obtained by nonequilibrium dynamics opens a new way to optimize quantum-probing strategies beyond ultracold atom systems, where our work already demonstrates a boost of sensitivity of roughly an order of magnitude. For ultracold atomic systems, the key elements for adopting our method are found in various mixture experiments: Broken time-reversal symmetry of endo- and exothermal SE rates is originating from different atomic Landé factors, and the need for individual quantum probes can be relaxed for other probe species, which lack strong probe-probe interactions; hence, the transient steady-state spin distribution of the probe can be established for sufficiently long times. Thereby, our method paves the way for thermometry in situations, where standard time-of-flight velocimetry in ultracold-gas experiments is notoriously delicate, including extremely low atomic densities, strongly interacting quantum gases, or quantum fluids in optical lattices.

Our specific system allows us to experimentally test fundamental relations of information theory and thermodynamics, such as Landauer's principle or the connection between Shannon entropy and Fisher information, out of equilibrium. In this work, the role of coherence is neglected, but our system may spark and test novel models to elucidate its role in the dynamics and its relation to Fisher information, which is an open question and under current investigation [36,37]. Moreover, reducing the bath size will allow us to follow the transition from a Markov to a non-Markov bath, shedding new light on the microscopic quantum dynamics for system-bath entanglement [38]. Finally, while we do not employ high-resolution imaging of the probes, our experimental system also paves the way to local probing of quantum gases or employing collective interaction effects [39].

\section{ACKNOWLEDGMENTS}

We thank Eric Lutz for helpful discussions. This work is funded in the early stage by the European Union via ERC Starting Grant "QuantumProbe" and in the final stage by Deutsche Forschungsgemeinschaft via Sonderforschungsbereich (SFB) SFB/TRR185, Project No. 277625399.

\section{APPENDIX A: EXPERIMENTAL PROCEDURE}

The $\mathrm{Rb}$ cloud is prepared by loading a laser-cooled cloud into a crossed dipole trap at $\lambda=1064 \mathrm{~nm}$. Changing the final dipole trap depth at the end of the evaporation, we can create $\mathrm{Rb}$ clouds with typically $N=5-9 \times 10^{3}$ atoms and temperatures between 0.2 and $1 \mu \mathrm{K}$. The dipole trap is then adiabatically compressed to a fixed final trap depth, yielding trap frequencies in radial and axial directions of $\omega_{r}=2 \pi \times 330 \mathrm{~Hz}$ and $\omega_{z}=2 \pi \times 50 \mathrm{~Hz}$, respectively, and atomic densities on the order of $10^{12}-10^{13} \mathrm{~cm}^{-3}$. The $\mathrm{Rb}$ cloud is then transferred into the insensitive magnetic field state $m_{F, \mathrm{Rb}}=0$ by microwave sweeps. Subsequently, few Cs atoms are captured in a high-gradient magneto-optical trap and loaded into an independent crossed dipole trap located $160 \mu \mathrm{m}$ from the $\mathrm{Rb}$ cloud. Cs atoms are further cooled down with a degenerate Raman sideband cooling scheme [40], pumping the Cs atoms in their absolute ground state $m_{F, \mathrm{Cs}}=3$. Thereafter, the Cs atoms are transferred into the desired internal state $m_{F, \mathrm{Cs}}=2$ by microwave-driven Landau-Zener transitions, near resonant to the hyperfine transition $(h \times 9.1 \mathrm{GHz})$. The use of a few Cs atoms (six on average) is a compromise between neglecting Cs-Cs interactions [41] and minimizing the influence on the bath on the one hand and obtaining sufficient statistics on the other hand. The limit of single probes, however, is routinely possible. Finally, Cs atoms are guided by the dipole trap potential into the ultracold cloud before the interaction starts. Because of the favorable ratio of mass and dipole force, Cs atoms experience almost the same trapping frequencies as $\mathrm{Rb}$ atoms. The magnetic field amplitude $B$ during the Cs-Rb interaction is calibrated with $\mathrm{Rb}$ atoms, using the $h \times 6.8 \mathrm{GHz}$ microwave 
transition that is resonant with the ground-level hyperfine splitting. This calibration allows us to control $B$ with an accuracy of $\pm 2 \mathrm{mG}$. Moreover, we take care to ramp up the magnetic field adiabatically in order to avoid mixing Zeeman states. After an interaction time, the Rb cloud is removed from the trap by a resonant laser pulse. The populations of Cs atoms in the different $m_{F, \text { Cs }}$ states are then inferred by a combination of state-sensitive microwave transitions at $h \times 9.1 \mathrm{GHz}$ and a hyperfine sensitive pushout laser pulse [42].

\section{APPENDIX B: FRACTION OF CS ATOMS UNDERGOING AN ENDOERGIC PROCESS}

During an endoergic SE collision, the Cs atom delivers $\Delta E / 2$ of energy, where $\Delta E / 2$ is the Zeeman energy splitting of the Cs atom [see Fig. 2(a)]. Operating at low magnetic field $B$, the splitting writes $\Delta E / 2=g_{F} \mu_{B} B$, where $g_{F}$ is the Lande factor $\left(g_{F}=1 / 4\right), \mu_{B}$ the Bohr magneton, and $B$ the magnetic field. However, for the endoergic collision to occur, the $\mathrm{Rb}$ atom requires $\Delta E$ of energy. As a consequence, $\Delta E / 2$ is lacking, which must be provided by the kinetic energy of the collision, which is given by $E_{c}=\mu v_{\text {rel }}^{2} / 2$, where $\mu$ is the reduced mass of $\mathrm{Rb}$ and $\mathrm{Cs}$ and $v_{\text {rel }}$ their relative velocity. Assuming that $\mathrm{Cs}$ and $\mathrm{Rb}$ atoms are thermalized at temperature $T$, the collision energy $E_{c}$ follows a Maxwell-Boltzmann distribution [43]

$$
p\left(E_{c}\right)=\left(\frac{1}{\pi k_{B} T}\right)^{3 / 2} 2 \pi \sqrt{E_{c}} \exp \left(-\frac{E_{c}}{k_{B} T}\right)
$$

where $k_{B}$ is the Boltzmann constant. The fraction of Cs atoms allowing a $\mathrm{SE}$ is thus given by $p(B, T)=$ $\int_{\Delta E / 2}^{\infty} p\left(E_{c}\right) d E_{c}$ and writes

$$
p(B, T)=1+\sqrt{\frac{\mu_{B} B}{\pi k_{B} T}} \exp \left(-\frac{\mu_{B} B}{4 k_{B} T}\right)-\operatorname{erf}\left(-\sqrt{\frac{\mu_{B} B}{4 k_{B} T}}\right),
$$

where erf is the error function. This is the expression used to plot Fig. 2(c) in the main text.

\section{APPENDIX C: SE SCATTERING CROSS SECTIONS}

The interaction between the $\mathrm{Rb}$ and $\mathrm{Cs}$ atoms is modeled by the interparticle singlet and triplet potentials. It allows for elastic and spin-exchange collisions [22]. Elastic collisions preserve the internal states of both collision partners after the collisions, leading to thermalization. SE processes lead to a spin transfer while maintaining the total magnetization $M=m_{F, \mathrm{Rb}}+m_{F, \mathrm{Cs}}$. Thus, only exoergic and endoergic processes with a spin transfer $\Delta m_{F, \mathrm{Cs}}= \pm 1$ for $\mathrm{Cs}$ and $\Delta m_{F, \mathrm{Rb}}=\mp 1$ for $\mathrm{Rb}$ are possible, where $\Delta m_{F, \mathrm{Cs}}<\Delta m_{F, \mathrm{Rb}}$ gives an exoergic process and $\Delta m_{F, \mathrm{Cs}}>\Delta m_{F, \mathrm{Rb}}$ an endoergic process. Scattering cross sections for the respective SE processes are calculated in a coupled-channel scattering model. The calculations are based on a $\mathrm{Cs}-\mathrm{Rb}$ interaction potential model obtained from more than $30 \times 10^{3}$ spectroscopy lines and Feshbach resonances [26]. Each individual calculation uses a fixed magnetic field $B$ and a fixed collision energy $E_{c}$. They are performed for all possible asymptotic channels $\left|m_{F, \mathrm{Cs}}, m_{F, \mathrm{Rb}}\right\rangle$. In Fig. 6, we show the calculated cross section $\sigma\left(B, E_{c}\right)$ of the endoergic and exoergic process for Cs atoms initially in $m_{F, \mathrm{Cs}}=2$ and $\mathrm{Rb}$ in $m_{F, \mathrm{Rb}}=0$. Additionally, we take into account the effect of the finite temperature in the cross section by calculating $\sigma(B, T)=\int p\left(E_{c}\right) \sigma\left(B, E_{c}\right) d E_{c}$, where $p\left(E_{c}\right)$ is defined in Eq. (B1). In Fig. 7, we plot the finite-temperature cross sections of both SE processes for Cs atoms initially in $m_{F, \mathrm{Cs}}=2$ and $\mathrm{Rb}$ in $m_{F, \mathrm{Rb}}=0$.
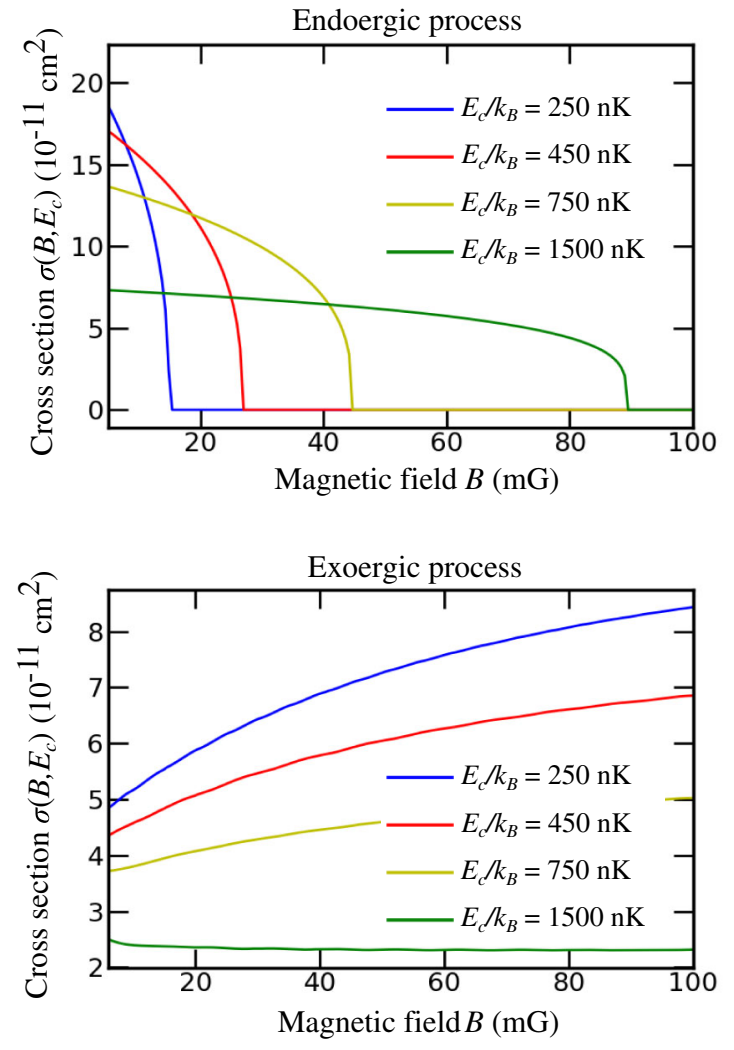

FIG. 6. Scattering cross sections for endoergic SE (top) and exoergic SE (bottom) for Cs in the state $m_{F, \mathrm{Cs}}=2$ and $\mathrm{Rb}$ in the state $m_{F, \mathrm{Rb}}=0$. The cross sections are plotted for four different fixed collision energies $E_{c}(250,450,750$, and $1500 \mathrm{nK})$. In the endoergic SE, the energy condition is underlined: If $E_{c} \leq \mu_{B} B / 4$, the collision is forbidden, and therefore, the cross section drops to 0 . 

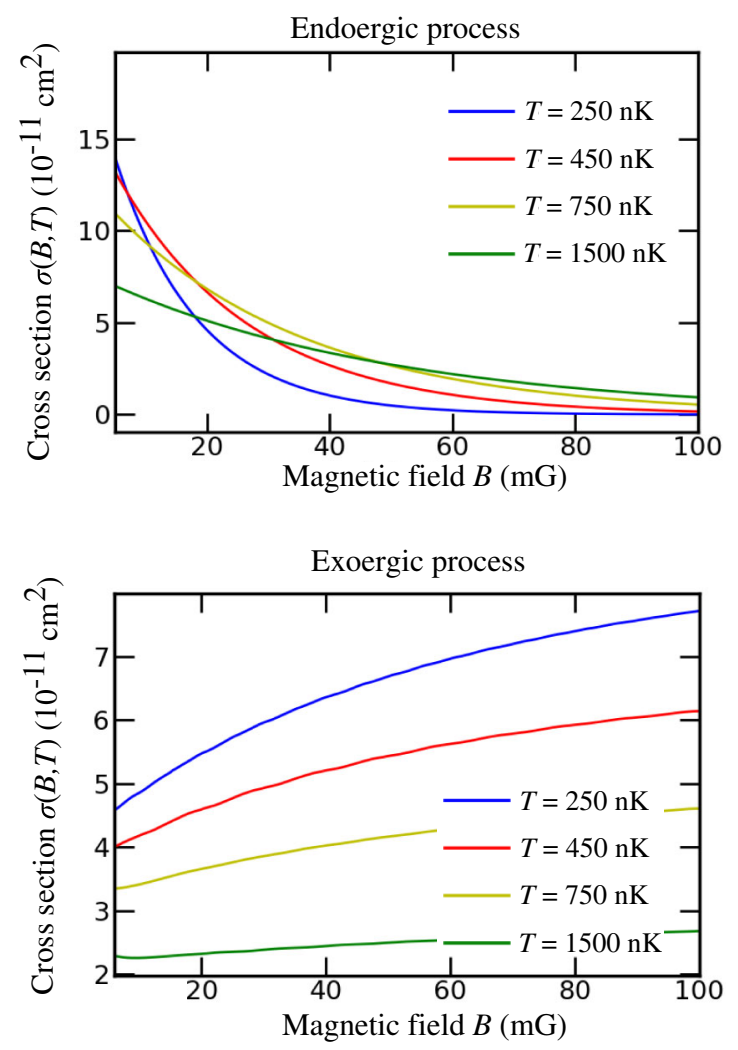

FIG. 7. Scattering cross sections for endoergic SE (top) and exoergic SE (bottom) for $\mathrm{Cs}$ in the state $m_{F, \mathrm{Cs}}=2$ and $\mathrm{Rb}$ in $m_{F, \mathrm{Rb}}=0$. The cross sections shown here include the effect of finite temperature and are plotted for four different temperatures $T(250,450,750$, and $1500 \mathrm{nK})$.

\section{APPENDIX D: SPIN-EXCHANGE MODEL}

The spin population of the Cs atoms immersed in the $\mathrm{Rb}$ cloud $\left(F_{\mathrm{Rb}}=1, m_{F, \mathrm{Rb}}=0\right)$ is governed by the endoergic and exoergic SE process. We model the population $N_{m_{F, C s}}$ of the seven internal states of the Cs atoms $\left(m_{F, \text { Cs }} \in[-3,-2,-1,0,1,2,3]\right)$ by a rate model. Each $m_{F, \mathrm{Cs}}$ spin state decays, on the one hand to the $m_{F, \mathrm{Cs}}+$ 1 state at the rate $\Gamma^{m_{F} \rightarrow m_{F}+1}$ due to the endoergic SE process and, on the other hand, to the $m_{F, \mathrm{Cs}}-1$ state at a rate $\Gamma^{m_{F} \rightarrow m_{F}-1}$ due to the exoergic SE process (see Fig. 8). In the meantime, each $m_{F, \mathrm{Cs}}$ spin state gains population from $m_{F, \text { Cs }}-1$ at the rate $\Gamma^{m_{F}-1 \rightarrow m_{F}}$ due to endoergic SE process and from $m_{F, \mathrm{Cs}}+1$ at the rate $\Gamma^{m_{F}+1 \rightarrow m_{F}}$ due to exoergic SE process (see also Fig. 8). It translates into the following differential equation for each $N_{m_{F, C s}}$ spin-state population

$$
\begin{aligned}
\dot{N}_{m_{F, \mathrm{CS}}}= & +\Gamma^{m_{F}+1 \rightarrow m_{F}} N_{m_{F, \mathrm{Cs}}+1}+\Gamma^{m_{F}-1 \rightarrow m_{F}} N_{m_{F, \mathrm{Cs}}-1} \\
& -\left(\Gamma^{m_{F} \rightarrow m_{F}-1}+\Gamma^{m_{F} \rightarrow m_{F}+1}\right) N_{m_{F}, \mathrm{Cs}} .
\end{aligned}
$$

In order to solve these differential equations, the different collision rates $\Gamma_{i}$ have to be inferred (six for the endoergic process and six for the exoergic process). They are given by

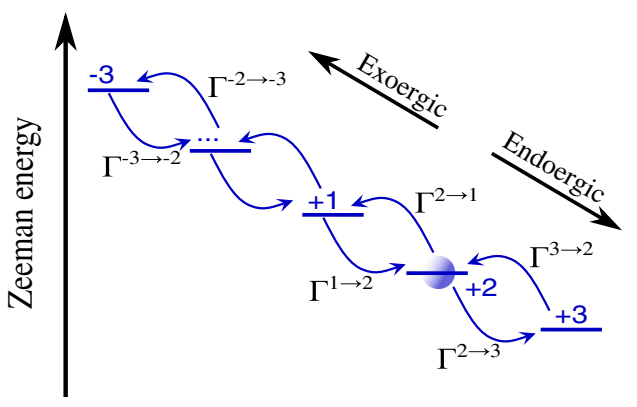

FIG. 8. Sketch of the rate model used for calculating the spin dynamic. For each spin state $m_{F, \text { Cs }}$, endoergic processes lead to a decay to the $m_{F, \mathrm{Cs}}+1$ state and a gain from the $m_{F, \mathrm{Cs}}-1$ state. Likewise, exoergic SE processes lead to a decay to the $m_{F, \mathrm{Cs}}-1$ state and a gain from the $m_{F, \text { Cs }}+1$ state.

$$
\Gamma_{i}=\langle n\rangle \sigma_{i}(B, T) \bar{v}
$$

where $\langle n\rangle$ is the Cs- $\mathrm{Rb}$ density overlap, $\sigma_{i}(B, T)$ the scattering cross section (which depends on the considered state), and $\bar{v}$ the relative velocity between $\mathrm{Rb}$ and Cs atoms. To calculate these three parameters, we first assume full thermalization of the $\mathrm{Cs}$ atoms in the $\mathrm{Rb}$ bath at temperature $T$. The thermalization rate $\Gamma_{\text {ther }}$ of a Cs atom is given by [44]

$$
\Gamma_{\text {ther }}=\frac{\Gamma_{\mathrm{el}}}{3} \xi \frac{N_{\mathrm{Rb}}+N_{\mathrm{Cs}}}{N_{\mathrm{Rb}}},
$$

where $\Gamma_{\mathrm{el}}$ is the scattering elastic collision rate and $\xi=\left[\left(4 m_{\mathrm{Rb}} m_{\mathrm{Cs}}\right) /\left(m_{\mathrm{Rb}}+m_{\mathrm{Cs}}\right)^{2}\right]$ the reduction factor for momentum exchange in a Cs-Rb collision due to the mass imbalance. The thermalization of a single $\mathrm{Cs}$ atom in a large $\mathrm{Rb}$ bath $\left(N_{\mathrm{Cs}} \ll N_{\mathrm{Rb}}\right)$ yields a thermalization rate of $\Gamma_{\text {ther }} \approx \Gamma_{\text {el }} / 3$. Since the elastic rate is 10 times higher than the SE rates, the thermalization of the Cs atom is always ensured at the moment of the SE collisions. As a consequence, the relative velocity between $\mathrm{Rb}$ and $\mathrm{Cs}$ writes

$$
\bar{v}=\sqrt{\frac{8 k_{B} T}{\pi \mu}},
$$

where $\mu$ is the reduced mass. The density-density overlap $\langle n\rangle$ of $\mathrm{Cs}$ and $\mathrm{Rb}$ at density $n_{\mathrm{Cs}}$ and $n_{\mathrm{Rb}}$ is

$$
\langle n\rangle=\int n_{\mathrm{Cs}}(\vec{r}) n_{\mathrm{Rb}}(\vec{r}) d \vec{r}
$$

and it is calculated assuming a Maxwell-Boltzmann distribution for $\mathrm{Cs}$ and $\mathrm{Rb}$. Finally, the different scattering cross sections are averaged over a thermalized distribution $\sigma_{i}(B, T)=\int p\left(E_{c}\right) \sigma_{i}\left(B, E_{c}\right) d E_{c}$, as we explain in the previous section.

Starting with an initial Cs population in the $m_{F, \text { Cs }}=2$ state, we numerically integrate Eq. (D1) and find excellent 


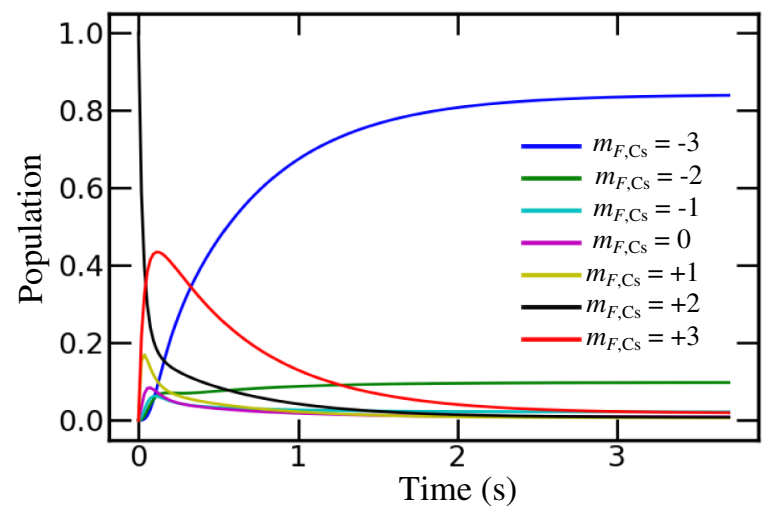

FIG. 9. Simulation of the spin-states' dynamics of Cs immersed in a Rb bath with $N_{\mathrm{Rb}}=7 \times 10^{3}$ atoms at $T=400 \mathrm{nK}$ and $B=$ $10 \mathrm{mG}$ [expected endoergic fraction $p(B, T)=0.84$ ]. The seven spin states $\left(m_{F, \mathrm{Cs}} \in[-3,-2,-1,0,1,2,3]\right)$ are plotted. The steady state is reached beyond 3 -s interaction time.

agreement between the theory and the experimental data, as we illustrate in Fig. 3(a) in the main text. Moreover, we also simulate with our model the Cs spin population with $N_{\mathrm{Rb}}=7 \times 10^{3}$ atoms at $T=400 \mathrm{nK}$ and $B=10 \mathrm{mG}$, which are typical numbers in our experiment. The result is plotted in Fig. 9. We observe that the steady state is reached after an interaction time of almost $3 \mathrm{~s}$, which leads to a non-negligible loss of $\mathrm{Cs}$ atoms due to three-body recombination $(\mathrm{Rb}-\mathrm{Rb}-\mathrm{Cs})$. This loss rate writes $\Gamma_{3 \text { body }}=$ $L_{3}\left\langle n^{2}\right\rangle$, with $\left\langle n^{2}\right\rangle=\int n_{\mathrm{Rb}}^{2}(\vec{r}) n_{\mathrm{Cs}}(\vec{r}) d \vec{r}$ and $L_{3}=28(1) \times$ $10^{-26} \mathrm{~Hz} \mathrm{~cm}^{-6}$ [21]. The expected value of the rate of three-body losses is $\Gamma_{3 \text { body }}=0.66 \mathrm{~Hz}$, leading to an expected lifetime of Cs $\tau=1 / \Gamma_{3 \text { body }}=1.5 \mathrm{~s}$. Therefore, a large fraction of Cs atoms should be lost when the steady state is reached in our system.

\section{APPENDIX E: MEAN AND FLUCTUATION OF ENERGY, PROBE ENTROPY, AND NUMBER OF SPIN COLLISIONS}

We denote each of the seven Cs spin-state populations with quantum number $m_{F} \in[3,2,1,0,-1,-2,-3]$ as $P_{m_{F}}$ with $\sum_{m_{F}} P_{m_{F}}=1$. From the modeled quantum-state distributions, we can extract useful observables such as the mean energy $\langle E\rangle$, the variance of energy $\left\langle E^{2}\right\rangle$, the entropy $S$ of the Cs atom's spin population, and the number of spin collisions. $\langle E\rangle$ is defined as

$$
\langle E\rangle=\sum_{m_{F}=+3}^{m_{F}=-3} P_{m_{F}} E_{m_{F}},
$$

where $E_{m_{F}}=\left(3-m_{F}\right) \Delta E / 2$, such as the energy of the ground state $\left|m_{F}=3\right\rangle$ is set to zero in our model. In the same way, $\left\langle E^{2}\right\rangle$ writes as

$$
\left\langle E^{2}\right\rangle=\sum_{m_{F}=+3}^{m_{F}=-3} P_{m_{F}} E_{m_{F}}^{2} .
$$

The fluctuations of energy are then given by $\sigma_{E}^{2}=$ $\left\langle E^{2}\right\rangle-\left\langle E^{2}\right\rangle$. Finally, the entropy $S$ is expressed as

$$
S=-k_{B} \sum_{m_{F}=+3}^{m_{F}=-3} P_{m_{F}} \log \left(P_{m_{F}}\right) \text {. }
$$

An example of the time evolution of entropy $S$ is depicted in Fig. 10(a). At $t=0$, we prepare all the Cs atoms in $m_{F}=2$, a zero-entropy state. Here we are maximally sensitive to any changes. The only constraint for the Cs system is that $\sum_{m_{F}} P_{m_{F}}=1$ (the energy of the Cs atoms $\langle E\rangle$

(a)
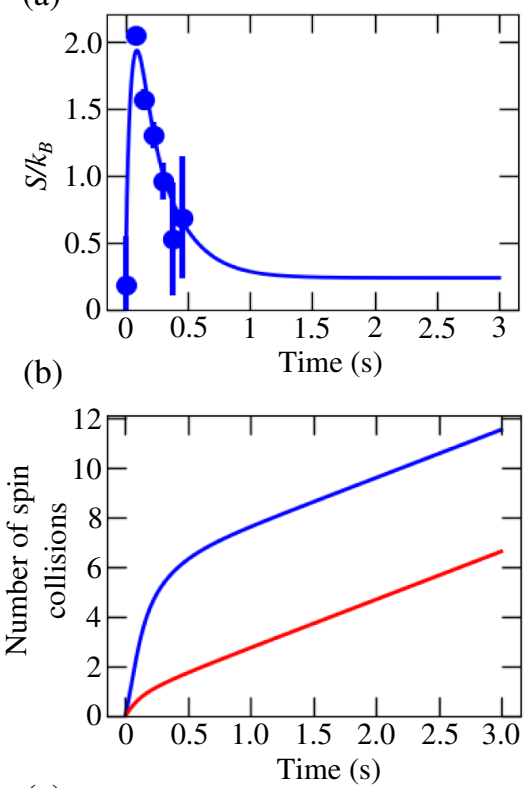

(c)

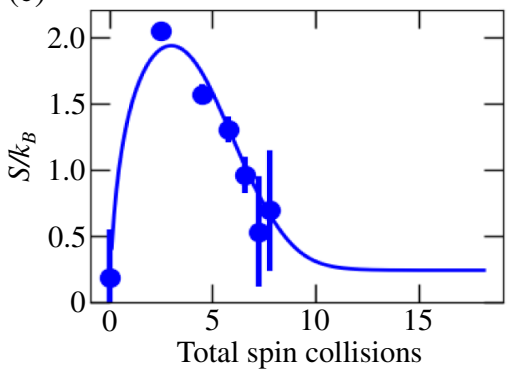

FIG. 10. Evolution of the entropy $S$ of the probe. The Cs atoms are initially prepared in $m_{F, \mathrm{Cs}}=2$, immersed in a $\mathrm{Rb}$ bath at $T=366 \mathrm{nK}$ with $N_{\mathrm{Rb}}=6.7 \times 10^{3}$ atoms. The magnetic field is $B=25 \mathrm{mG}$. Experimental points (circle) and theoretical predictions (solid lines) are shown. (a) Time evolution of the entropy of the probe $S$. (b) Time evolution of the mean number of spin collisions: in red the mean number of endoergic spin collisions and in blue the mean number of exoergic spin collisions. (c) The entropy of the probe as a function of the total number of spin collisions is shown. 
is not conserved but coupled to the $\mathrm{Rb}$ bath). Using the Lagrange multiplier with the Lagrangian function $L\left(P_{m_{F}}, \lambda\right)=S-\lambda\left(\sum_{m_{F}} P_{m_{F}}-1\right)$, we find that the entropy is theoretically maximized when the distribution population of $\mathrm{Cs}$ atoms is equally spread (i.e., $P_{m_{F}}=1 / 7$ ). Experimentally, we find that a maximum entropy is reached for $t=90 \mathrm{~ms}$, where we are closest to this situation. It indicates that the nonequilibrium spin distribution can yield much better information than the steady-state observation. In order to quantify the number of spin collisions necessary to reach the optimum, we first calculate the mean spin collision rate $\langle\Gamma(t)\rangle$,

$$
\begin{aligned}
\langle\Gamma(t)\rangle= & \left\langle\Gamma_{\text {endo }}(t)\right\rangle+\left\langle\Gamma_{\text {exo }}(t)\right\rangle \\
= & \sum_{m_{F}=+2}^{m_{F}=-3} P_{m_{F}} \Gamma_{\text {endo }}^{m_{F} \rightarrow m_{F}+1} \\
& +\sum_{m_{F}=+3}^{m_{F}=-2} P_{m_{F}} \Gamma_{\text {exo }}^{m_{F} \rightarrow m_{F}-1} .
\end{aligned}
$$

The number of spin collisions $N_{\text {spin }}=N_{\text {endo }}+N_{\text {exo }}$ is then deduced by integrating Eq. (E4)

$$
\begin{aligned}
N_{\text {spin }}(t) & =N_{\text {endo }}+N_{\text {exo }} \\
& =\int_{0}^{t}\left(\left\langle\Gamma_{\text {endo }}\left(t^{\prime}\right)\right\rangle+\left\langle\Gamma_{\text {endo }}\left(t^{\prime}\right)\right\rangle\right) d t^{\prime} .
\end{aligned}
$$

An example of time evolution of endoergic and exoergic spin collisions is represented in Fig. 10(b). We can then track the entropy of the probe in the function of the number of spin collisions [Fig. 10(c)]: We find that the maximum of the entropy is obtained for a mean of only three spin-exchange collisions (on average 2.5 exoergic and 0.5 endoergic spinexchange collisions).

\section{APPENDIX F: STEADY STATE}

In the steady state, the temperature depends only on the scattering cross section $\sigma_{i}(B, T)$ since all the rates $\Gamma_{i}$ have the same dependence in regards to the density $\langle n\rangle$ and the relative velocity $\bar{v}$ [Eq. (D2)]. Therefore, thermometry can also be performed using the steady state. To demonstrate this, we investigate the fluctuation of energy $\sigma_{E}$ to the steady state for different temperatures. The populations are inferred by solving Eq. (D1) with $\dot{N}_{m_{F . \mathrm{Cs}}}=0$, and replacing all the rates $\Gamma_{i}$ by the corresponding cross section $\sigma_{i}(B, T)$. Figure 11 shows the behavior of $\sigma_{E}$ with the temperature for different magnetic fields $B$. If the thermal energy is significantly larger than the Zeeman energy and thus the fraction of endoergic SE amounts to more than a few percent according to Eq. (B2), we observe a linear behavior of the distribution's width with the temperature $T$. Furthermore, we observe that the proportionality constant is independent of the magnetic field.
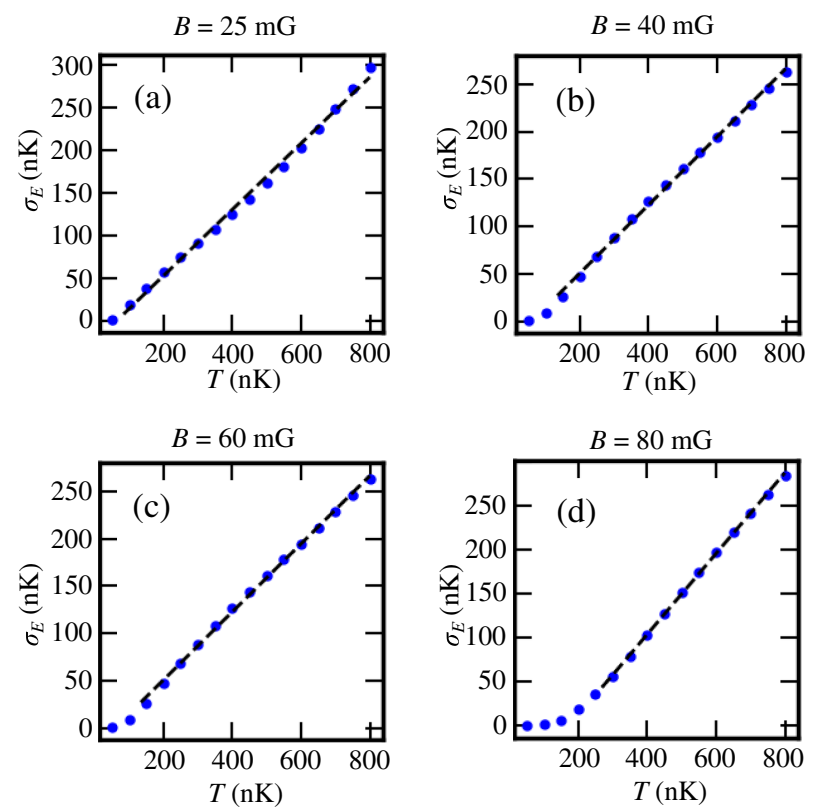

FIG. 11. Fluctuation of energy $\sigma_{E}$ of the steady state of the spin population of the probe as a function of the temperature $T$ for a fixed magnetic field $B$ : (a) $B=25 \mathrm{mG}$, (b) $B=40 \mathrm{mG}$, (c) $B=60 \mathrm{mG}$, (d) $B=80 \mathrm{mG}$. The dots are the theoretical points, and the dashed lines represent the linear fit.

\section{APPENDIX G: DETERMINATION OF SPIN TEMPERATURES AND SPIN MAGNETIC FIELDS}

To extract the temperature or the magnetic field from the spin population of the probe, we perform a $\chi^{2}$ analysis. For each measurement comprising the seven internal states $m_{F, \text { exp }} \in[-3,-2,1,0,1,2,3]$ of the Cs atom, a reduced $\chi_{\nu}^{2}$ is calculated

$$
\chi_{\nu}^{2}(\theta)=\frac{1}{\nu} \sum_{m_{F}} \frac{\left[P_{m_{F, \text { exp }}}-P_{m_{F, \text { theo }}}(\theta)\right]^{2}}{\sigma_{m_{F, \text { exp }}}^{2}},
$$

where $\theta=T$ or $B, P_{m_{F, \text { exp }}}$ the measured populations associated with the experimental error bars $\sigma_{m_{F, \text { exp }}}$. $P_{m_{F, \text { theo }}}(\theta)$ are the theoretical populations deduced from our microscopic model, where only the parameter $\theta$ is a free parameter. Finally, $\nu$ is the number of degrees of freedom, which is 7 in our case. An example of a $\chi^{2}$ analysis is shown in Fig. 12. We extract the temperature $T_{\text {spin }}$ (respectively, the magnetic field $B_{\text {spin }}$ ) by finding the minimum of $\chi_{\nu}^{2}(T)$ [respectively, $\chi_{\nu}^{2}(B)$ ]. The error bar corresponds to the value of the parameter of interest $\theta=T$ or $B$ if we increase $\chi_{\nu}^{2}(\theta)$ by 1 , translating to 1 standard deviation $\sigma$ in the error bar [28]. In addition, we also study the systematic deviation of the spin temperatures $T_{\text {spin }}$ due to the uncertainty of $\pm 2 \mathrm{mG}$ on the magnetic field $B$. Including this effect in the $\chi^{2}$ analysis, we find a systematic error close to $30 \mathrm{nK}$ for the spin temperatures. 

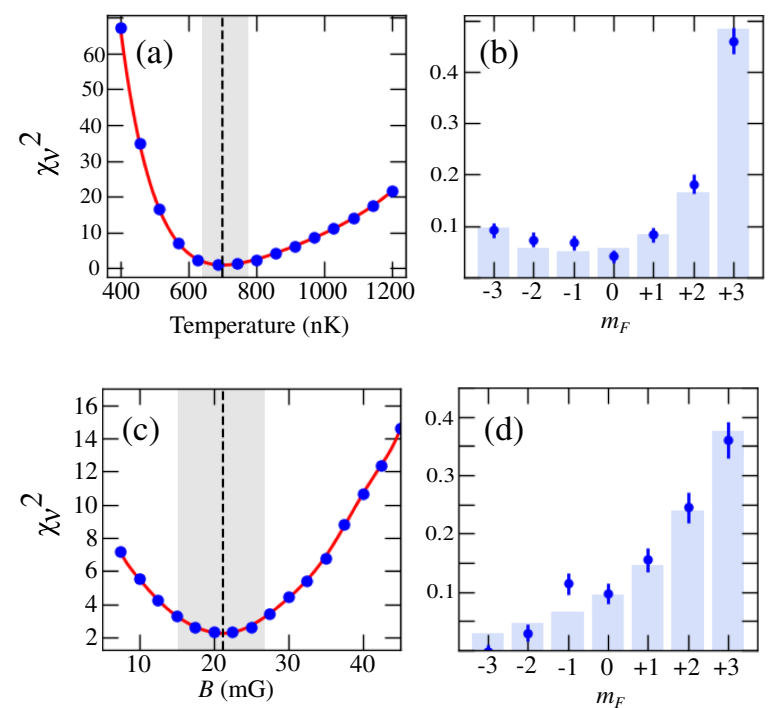

FIG. 12. Extraction of the spin temperature $T_{\text {spin }}$ and the spin magnetic field $B_{\text {spin }}$ with a $\chi^{2}$ analysis. (a) shows a $\chi_{\nu}^{2}(T)$ curve (the magnetic field is fixed at $B=10 \mathrm{mG}$ ) and (c) represents a $\chi_{\nu}^{2}(B)$ curve (the temperature is fixed at $T=1 \mu K$ ). In each curve, the vertical dashed line represents the minimum value of the $\chi_{\nu}^{2}$, which marks the extracted $T_{\text {spin }}$ or $B_{\text {spin }}$. The slightly gray band represents the standard deviation corresponding to an increase of $\chi_{\nu}^{2}$ of 1 . We find $T_{\text {spin }}=702_{-76}^{+60}$ for (a) and $B_{\text {spin }}=21.6_{-6.1}^{+5.7}$ for (c). (b) and (d) represent the measured populations (dots) and the best parameter of interest with the model (histogram).

\section{APPENDIX H: BURES DISTANCE AND FISHER INFORMATION}

The investigation of the thermal and magnetic sensitivity of our probe is done using the mathematical framework of the quantum Fisher information. The thermal sensitivity means that the temperature $T$ is varied, but the magnetic field $B$ is constant. On the contrary, the magnetic sensitivity means that $B$ is varied but $T$ is constant. Neglecting the coherences in the system, we describe each state by a diagonal density matrix $\hat{\rho}(B, T)=\sum_{m_{F}} P_{m_{F}}(B, T)\left|m_{F}\right\rangle\left\langle m_{F}\right|$, where $P_{m_{F}}(B, T)$ are the spin populations of the probe at $T$ and $B$. We denote the parameter of interest as $\theta$ (here $\theta=B$ or $T$ ). We quantify the distance between two quantum states at $\theta$ and $\theta+\delta \theta$ using the Bures distance as [45]

$$
\begin{aligned}
d_{\text {Bures }}^{2}(\delta \theta)= & 2-2 \operatorname{tr}(\sqrt{\sqrt{\hat{\rho}(\theta+\delta \theta)} \hat{\rho}(\theta) \sqrt{\hat{\rho}(\theta+\delta \theta)}}) \\
= & 2-2 \sum_{m_{F}}\left[P_{m_{F}}(\theta) P_{m_{F}}(\theta+\delta \theta)\right]^{1 / 2} .
\end{aligned}
$$

The latter expression uses the fact that the density matrix is diagonal, and thus, the density operators between the original and modified quantum state commute. In these conditions, the Bures distance coincides with the so-called Hellinger distance. The relation between the Bures distance and the quantum Fisher information $F_{\theta}$ is [29]
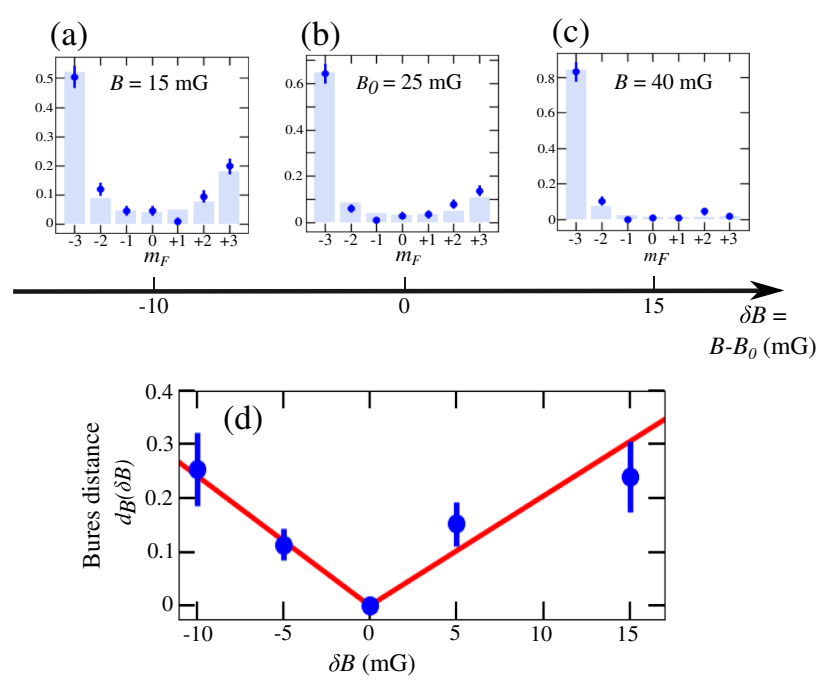

FIG. 13. Experimental extraction of the quantum Fisher information. The temperature is fixed at $T=280 \mathrm{nK}$. In (a)-(c), we represent the spin populations of the Cs atoms for different magnetic fields $B$ : The dots are the experimental points, and the histograms are the theoretical expectation values. In (d), we represent the Bures distance between the different states centered at $B_{0}=25 \mathrm{mG}$. In red, the Bures distance is inferred by the theoretical spin populations and in blue by the experimental populations.

$$
d_{\text {Bures }}(\delta \theta)=\sqrt{F_{\theta}} \delta \theta+\mathcal{O}\left(\delta \theta^{2}\right) .
$$

In Fig. 13(d), we represent the Bures distance when $\theta=B$. We observe a linear behavior of the Bures distance-the slopes thus representing the Fisher information-that we refer to as the sensitivity. More precisely, we perform two Taylor expansions: one for $\delta \theta<0$ and one for $\delta \theta>0$. In general, these two Taylor expansions are equal since the system considered is symmetric and $d_{\text {Bures }}^{2}(\delta \theta)$ is directly analyzed [32]. However, in our case, due to the broken symmetry between endoergic and exoteric processes, these quantities are slightly different (between $10 \%$ and 20\%). Nonetheless, they share the same behavior. Hence, there is no additional information gained from studying them separately, and we simply study the mean value.

[1] G. Kucsko, P. C. Maurer, N. Y. Yao, M. Kubo, H. J. Noh, P. K. Lo, H. Park, and M. D. Lukin, Nanometre-Scale Thermometry in a Living Cell, Nature (London) 500, 54 (2013).

[2] F. Haupt, A. Imamoglu, and M. Kroner, Single Quantum Dot as an Optical Thermometer for Millikelvin Temperatures, Phys. Rev. Applied 2, 024001 (2014).

[3] S. Kotler, N. Akerman, Y. Glickman, A. Keselman, and R. Ozeri, Single Ion Quantum Lock-In Amplifier, Nature (London) 473, 61 (2011).

[4] C. L. Degen, F. Reinhard, and P. Cappellaro, Quantum Sensing, Rev. Mod. Phys. 89, 035002 (2017). 
[5] T. H. Johnson, S. R. Clark, M. Bruderer, and D. Jaksch, Impurity Transport through a Strongly Interacting Bosonic Quantum Gas, Phys. Rev. A 84, 023617 (2011).

[6] L. A. Correa, M. Mehboudi, G. Adesso, and A. Sanpera, Individual Quantum Probes for Optimal Thermometry, Phys. Rev. Lett. 114, 220405 (2015).

[7] R. Olf, F. Fang, G. E. Marti, A. MacRae, and D. M. Stamper-Kurn, Thermometry and Cooling of a Bose Gas to 0.02 Times the Condensation Temperature, Nat. Phys. 11, 720 (2015).

[8] R. S. Lous, I. Fritsche, M. Jag, B. Huang, and R. Grimm, Thermometry of a Deeply Degenerate Fermi Gas with a Bose-Einstein Condensate, Phys. Rev. A 95, 053627 (2017).

[9] F. M. Spiegelhalder, A. Trenkwalder, D. Naik, G. Hendl, F. Schreck, and R. Grimm, Collisional Stability of ${ }^{40} \mathrm{~K}$ Immersed in a Strongly Interacting Fermi Gas of ${ }^{6} \mathrm{Li}$, Phys. Rev. Lett. 103, 223203 (2009).

[10] M. Hohmann, F. Kindermann, T. Lausch, D. Mayer, F. Schmidt, and A. Widera, Individual Tracer Atoms in an Ultracold Dilute Gas, Phys. Rev. A 93, 043607 (2016).

[11] W. Ketterle, D. S. Durfee, and D. M. Stamper-Kurn,Making, Probing and Understanding Bose-Einstein Condensates, in Bose-Einstein Condensation in Atomic Gases, Volume Course CXL of Proceedings International of School of Physics "Enrico Fermi" (IOS Press, Amsterdam, 1999), pp. 67-176.

[12] C. W. Helstrom, Quantum Detection and Estimation Theory (Academic Press, New York, 1976).

[13] T. H. Johnson, F. Cosco, M. T. Mitchison, D. Jaksch, and S. R. Clark, Thermometry of Ultracold Atoms via Nonequilibrium Work Distributions, Phys. Rev. A 93, 053619 (2016).

[14] C. Degen, Microscopy with Single Spins, Nat. Nanotechnol. 3, 643 (2008).

[15] A. Rivas, S. Huelga, and M. Plenio, Quantum NonMarkovianity: Characterization, Quantification and Detection, Rep. Prog. Phys. 77, 094001 (2014).

[16] A. Retzker, J. I. Cirac, M. B. Plenio, and B. Reznik, Methods for Detecting Acceleration Radiation in a BoseEinstein Condensate, Phys. Rev. Lett. 101, 110402 (2008).

[17] R. Dorner, S. R. Clark, L. Heaney, R. Fazio, J. Goold, and V. Vedral, Extracting Quantum Work Statistics and Fluctuation Theorems by Single-Qubit Interferometry, Phys. Rev. Lett. 110, 230601 (2013).

[18] D. Hangleiter, M. T. Mitchison, T. H. Johnson, M. Bruderer, M. B. Plenio, and D. Jaksch, Nondestructive Selective Probing of Phononic Excitations in a Cold Bose Gas Using Impurities, Phys. Rev. A 91, 013611 (2015).

[19] A. M. Kaufman, B. J. Lester, and C. A. Regal, Cooling a Single Atom in an Optical Tweezer to Its Quantum Ground State, Phys. Rev. X 2, 041014 (2012).

[20] C. Zipkes, S. Palzer, C. Sias, and M. Kohl, A Trapped Single Ion inside a Bose-Einstein Condensate, Nature (London) 464, 388 (2010).

[21] D. Mayer, F. Schmidt, D. Adam, S. Haupt, J. Koch, T. Lausch, J. Nettersheim, Q. Bouton, and A. Widera, Controlled Doping of a Bosonic Quantum Gas with Single Neutral Atoms, J. Phys. B 52, 015301 (2018).
[22] F. Schmidt, D. Mayer, Q. Bouton, D. Adam, T. Lausch, J. Nettersheim, E. Tiemann, and A. Widera, Tailored SingleAtom Collisions at Ultralow Energies, Phys. Rev. Lett. 122, 013401 (2019).

[23] F. Pobell, Matter and Methods at Low Temperatures (Springer, Berlin, 2007).

[24] M. Fattori, T. Koch, S. Goetz, A. Griesmaier, S. Hensler, J. Stuhler, and T. Pfau, Demagnetization Cooling of a Gas, Nat. Phys. 2, 765 (2006).

[25] B. Naylor, E. Maréchal, J. Huckans, O. Gorceix, P. Pedri, L. Vernac, and B. Laburthe-Tolra, Cooling of a Bose-Einstein Condensate by Spin Distillation, Phys. Rev. Lett. 115, 243002 (2015).

[26] T. Takekoshi, M. Debatin, R. Rameshan, F. Ferlaino, R. Grimm, H. C. Nagerl, C. R. Le Sueur, J. M. Hutson, P. S. Julienne, S. Kotochigova, and E. Tiemann, Towards the Production of Ultracold Ground-State RbCs Molecules: Feshbach Resonances, Weakly Bound States, and the Coupled-Channel Model, Phys. Rev. A 85, 032506 (2012).

[27] C. E. Shannon, A mathematical theory of communication, Bell Syst. Tech. J. 27, 379 (1948).

[28] P. R. Bevington and D. K. Robinson, Data Reduction and Error Analysis for the Physical Sciences (McGraw-Hill, New York, 2003).

[29] S. L. Braunstein and C. M. Caves, Statistical Distance and the Geometry of Quantum States, Phys. Rev. Lett. 72, 3439 (1994).

[30] W. Wasilewski, K. Jensen, H. Krauter, J. J. Renema, M. V. Balabas, and E.S. Polzik, Quantum Noise Limited and Entanglement-Assisted Magnetometry, Phys. Rev. Lett. 104, 133601 (2010).

[31] J. M. Boss, K. S. Cujia, J. Zopes, and C. L. Degen, Quantum Sensing with Arbitrary Frequency Resolution, Science 356, 837 (2017).

[32] H. Strobel, W. Muessel, D. Linnemann, T. Zibold, D. B. Hume, L. Pezze, A. Smerzi, and M. K. Oberthaler, Fisher Information and Entanglement of Non-Gaussian Spin States, Science 345, 424 (2017).

[33] A. Evrard, V. Makhalov, T. Chalopin, L. A. Sidorenkov, J. Dalibard, R. Lopes, and S. Nascimbene, Enhanced Magnetic Sensitivity with Non-Gaussian Quantum Fluctuations, Phys. Rev. Lett. 122, 173601 (2019).

[34] S. Luo and Q. Zhang, Informational Distance on QuantumState Space, Phys. Rev. A 69, 032106 (2004).

[35] E. Clarkson, Relation between Bayesian Fisher Information and Shannon Information for Detecting a Change in a Parameter, J. Opt. Soc. Am. A 36, 1209 (2019).

[36] X. Feng and L. Wei, Quantifying Quantum Coherence with Quantum Fisher Information, Sci. Rep. 7, 15492 (2017).

[37] H. Kwon, K. Tan, S. Choi, and H. Jeong, Quantum Fisher Information on Its Own Is Not a Valid Measure of the Coherence, Results Phys. 9, 1594 (2018).

[38] H. P. Breuer, E.-M. Laine, and J. Piilo, Measure for the Degree of Non-Markovian Behavior of Quantum Processes in Open Systems, Phys. Rev. Lett. 103, 210401 (2009).

[39] M. Mehboudi, A. Lampo, C. Charalambous, L. A. Correa, M. A. Garcia-March, and M. Lewenstein, Using Polarons for Sub-nK Quantum Nondemolition Thermometry in a 
Bose-Einstein Condensate, Phys. Rev. Lett. 122, 030403 (2019).

[40] A. J. Kerman, V. Vuletic, C. Chin, and S. Chu, Beyond Optical Molasses: 3D Raman Sideband Cooling of Atomic Cesium to High Phase-Space Density, Phys. Rev. Lett. 84, 439 (2000).

[41] C. Chin, V. Vuletic, A. J. Kerman, S. Chu, E. Tiesinga, P. J. Leo, and C. J. Williams, Precision Feshbach Spectroscopy of Ultracold $\mathrm{Cs}_{2}$, Phys. Rev. A 70, 032701 (2004).

[42] F. Schmidt, D. Mayer, Q. Bouton, D. Adam, T. Lausch, N. Spethmann, and A. Widera, Quantum Spin Dynamics of
Individual Neutral Impurities Coupled to a Bose-Einstein Condensate, Phys. Rev. Lett. 121, 130403 (2018).

[43] M. Cannoni, Relativistic $\left\langle\sigma v_{\text {rel }}\right\rangle$ in the Calculation of Relics Abundances: A Closer Look, Phys. Rev. D 89, 103533 (2014).

[44] M. Mudrich, S. Kraft, K. Singer, R. Grimm, A. Mosk, and M. Weidemüller, Sympathetic Cooling with Two Atomic Species in an Optical Trap, Phys. Rev. Lett. 88, 253001 (2002).

[45] M. Hübner, Explicit Computation of the Bures Distance for Density Matrices, Phys. Lett. A 163, 239 (1992). 